\title{
What do the latest guidelines tell us about UTIs in children under 2 years of age
}

\author{
Kjell Tullus
}

Received: 27 October 2011 /Revised: 22 November 2011 / Accepted: 23 November 2011 /Published online: 28 December 2011

(C) IPNA 2011

\begin{abstract}
The American Academy of Pediatrics (AAP) recently published new guidelines on diagnosing and managing infants and children younger than 2 years who have had a febrile urinary tract infection (UTI). They recommend, as previously did the National Institute for Health and Clinical Excellence (NICE) in the UK, a marked reduction in the imaging that these children should undergo. Both NICE and AAP agree that prophylactic antibiotic treatment should not be routinely used in these children, even in those with major vesicoureteric reflux.
\end{abstract}

Keywords Guidelines · Febrile UTI · Infants · children · Imaging $\cdot$ Prophylactic antibiotics $\cdot$ NICE $\cdot$ AAP

\section{Introduction}

Over the last decade the management of children with a febrile urinary tract infection (UTI) has become quite controversial. The old model was that all children were investigated using ultrasound (US), some kind of nuclear imaging such as dimercaptosuccininc acid (DMSA), and a micturition cystourethrogram (MCUG). The aim of these diagnostic investigations was to identify all children with vesicoureteric reflux (VUR) or any uptake defect on the nuclear images. Children with VUR of any grade were treated with prophylactic antibiotics. It was also common practice for all children with a febrile UTI to be hospitalised for intravenous (IV) treatment with antibiotics. However, critical voices are now being heard on the

\section{K. Tullus $(\bowtie)$}

Pediatric Nephrology,

Great Ormond Street Hospital,

London WC1N 3JH, UK

e-mail: Kjell.Tullus@gosh.nhs.uk need for such extensive investigations and are advocating a more selective approach [1].

Four years ago NICE (the National Institute for Health and Clinical Excellence) in the UK presented new guidelines that challenged many of these old routines, thereby initiating a major debate among clinicians $[2,3]$. The most controversial proposal was the quite major reduction in how much imaging a child should undergo after a first febrile UTI. The American Academy for Pediatrics (AAP) has recently presented their revised guidelines [4]. This organisation has focused on the most important group, children between the age of 2 months and 2 years with a febrile UTI, and most of the recommendations apply to children with a first time UTI. These guidelines are written in quite a different format than those of NICE, but the recommendations of both organisations are in many ways quite similar. However, AAP gives the level of each evidence while this is not done by NICE.

Here I discuss these two guidelines, pointing out the similarities and differences between them and also emphasising the differences to the previous generation of guidelines.

\section{When to suspect a UTI}

It is emphasised both by NICE and AAP that the symptoms of a febrile UTI are very non-specific, particularly in small children. The clinician assessing a febrile infant with no apparent source for the fever should always consider the possibility of a UTI, and in all these children a urine sample should be obtained.

This is clearly not controversial, but the method of urine collection is. AAP emphasises that the urine sample needs to be obtained by either a bladder puncture or as a catheterised sample. It point outs that cultures from bags applied to the 
perineum have an unacceptably high false-positive rate and that they are valid only when they yield a negative result.

\section{Diagnosis}

The diagnosis of a UTI should, according to the recommendations of the AAP, be based both on a urinalysis showing pyuria and/or bacteriuria and a positive culture. The AAP discusses the difficulty of reaching a correct diagnosis of a symptomatic UTI in an infant with previous (but in most cases unknown) asymptomatic bacteriuria (ABU) and a concurrent febrile infection from another source. The AAP argues that children with $\mathrm{ABU}$ will have absent or lower leukocyte counts in their urine compared to children with a symptomatic infection. This important diagnostic difficulty is not addressed by NICE. An acute DMSA to evaluate renal involvement is not recommended in any of the guidelines.

For several decades, the accepted cut-off limit for a true UTI has been a bacterial count of more than $10^{5}$ bacteria per millilitre. AAP acknowledges that this definition is operational and not absolute and recommends that a lower threshold of $5 \times 10^{4}$ bacteria per millilitre should be used as this will increase the sensitivity of the culture but at the expense of decreased specificity.

\section{Treatment}

Both NICE and AAP agree that oral treatment works equally well as IV in infants and children that are not septic and who reliably can take the drug by mouth. Children who need IV treatment should be switched to oral medication as soon as feasible. Neither AAP nor NICE give any firm recommendations on which empiric antibiotic that should be used, but both point out that the choice should take into account local bacterial susceptibility patterns in collaboration with the local bacteriological laboratory. NICE recommends 10 days of treatment while AAP states that there is no scientific evidence for whether 7, 10 or 14 days are better.

\section{Ultrasound}

The AAP recommends that all small children who have had a febrile UTI should undergo a renal and bladder ultrasound (RBUS). NICE is more selective and recommends this approach only for infants younger than 6 months and for older children if they have an atypical or recurrent UTI. NICE provides a list of situations when a UTI should be regarded as atypical, including a seriously ill child, poor urine flow, abdominal or bladder mass, raised serum creatinine, septicaemia, failure to respond to treatment with antibiotics within $48 \mathrm{~h}$ and an infection with a non-Escherichia coli organism. AAP points out that the diagnostic yield from a RBUS is low; and that only $1-2 \%$ of all children will have actionable findings (according to the AAP) as the use of prenatal ultrasound has reduced the prevalence of previously unknown problems.

Both AAP and NICE recommend that when a RBUS is assessed to be necessary, it should be performed early, within a few days, only in a small subset of children, namely those with an atypical or very severe infection; the remainder should have the RBUS done later. NICE recommends this to be done within 6 weeks. NICE recommends the late US for mainly practical reasons, while AAP also points out that the results from a RBUS that is performed too early can be misleading and show acute changes that will resolve spontaneously.

\section{Other imaging}

Both AAP and NICE recommend radically reducing the number of further investigations. AAP recommends no more imaging in children with a first time acute febrile UTI if there are no additional features indicated by the RBUS, i.e. hydronephrosis, scarring or other signs of high grade VUR or urinary tract obstruction. NICE agrees and recommends a MCUG only in the youngest infants below the age of 6 month if they have had an atypical UTI as defined above. An MCUG is not recommended in slightly older infants, aged more than 6 months, even in the presence of an atypical infection. Further evaluation should, according to AAP, be performed after a recurrent infection, and NICE agrees on the need for a MCUG but only in those with recurrences below the age of 6 months.

Regarding nuclear imaging, typically DMSA, NICE recommends this be done 4-6 months after the acute infection in children younger than 3 years who have had a recurrent or atypical infection. AAP is much less impressed by the need to do a DMSA and states that this is not recommended as part of the routine evaluation of infants after their first febrile UTI. The AAP views nuclear scanning as a useful research approach.

\section{Prophylactic antibiotics}

The other radical treatment change that appears in both the NICE and AAP guidelines is the recommendation that prophylactic antibiotics should not be routinely used even in children with VUR. When the NICE guidelines were written, only a few years ago, there were no results available from good randomised controlled trials on the use of antibiotic prophylaxis in children who have had a UTI; thus, the only conclusion that could be made was that prophylaxis could not 
be routinely recommended. Since then six quite good studies have been performed [5-10], and the findings from these studies have been summarised in the publication by APP in a meta-analyses. This analysis shows that the use of antibiotic prophylaxis provided no benefit over placebo even in children with high grade VUR. Very few children with grade V VUR were, however, included in the studies. There is one problem with this meta-analysis, namely, boys and girls were analysed together. The results from the Swedish reflux study clearly showed that girls with VUR grade III-IV between the age of 2 and 4 years did benefit from the prophylactic treatment while boys had too few recurrent infections, even in the placebo group, for the researchers to identify a difference [5].

\section{Conclusion}

Both AAP and NICE have presented recommendations that radically reduce imaging procedures in children who have had a febrile UTI. Both organisations recommend more imaging after recurrent infections and in those children with atypical features of the infection. NICE is more precise than AAP in terms of defining an atypical infection. A major reduction in the use of prophylactic antibiotics is also endorsed in both the USA and UK. It seems that we now again have come to a time period with less controversy and more agreement on the management of these children.

\section{References}

1. Marks SD, Gordon I, Tullus K (2008) Imaging in childhood urinary tract infections: time to reduce investigations. Pediatr Nephrol 23:9-17
2. Coulthard MG (2007) NICE on childhood UTI: Nasty processes produce nasty guidelines. Br Med J 335:463-464

3. Mori R, Lakhanpaul M, Verrier-Jones K (2007) Diagnosis and management of urinary tract infection in children: summary of NICE guidance. Br Med J 335:395-397

4. Subcommittee on Urinary Tract Infection, Steering Committee on Quality Improvement and Management (2011) Urinary tract infection: clinical practice guideline for the diagnosis and management of the Initial UTI in febrile infants and children 2 to 24 months. Pediatrics. doi: 10.1542/peds.2011-1330

5. Brandstrom P, Neveus T, Sixt R, Stokland E, Jodal U, Hansson S (2010) The Swedish reflux trial in children: IV. Renal damage J Urol 184:292-297

6. Craig JC, Simpson JM, Williams GJ, Lowe A, Reynolds GJ, McTaggart SJ, Hodson EM, Carapetis JR, Cranswick NE, Smith G, Irwig LM, Caldwell PH, Hamilton S, Roy LP (2009) Antibiotic prophylaxis and recurrent urinary tract infection in children. $\mathrm{N}$ Engl J Med 361:1748-1759

7. Garin EH, Olavarria F, Garcia NV, Valenciano B, Campos A, Young L (2006) Clinical significance of primary vesicoureteral reflux and urinary antibiotic prophylaxis after acute pyelonephritis: a multicenter, randomized, controlled study. Pediatrics 117:626632

8. Montini G, Rigon L, Zucchetta P, Fregonese F, Toffolo A, Gobber D, Cecchin D, Pavanello L, Molinari PP, Maschio F, Zanchetta S, Cassar W, Casadio L, Crivellaro C, Fortunati P, Corsini A, Calderan A, Comacchio S, Tommasi L, Hewitt IK, Da DL, Zacchello G, Dall'amico R (2008) Prophylaxis after first febrile urinary tract infection in children? A multicenter, randomized, controlled, noninferiority trial. Pediatrics 122:1064-1071

9. Pennesi M, Travan L, Peratoner L, Bordugo A, Cattaneo A, Ronfani L, Minisini S, Ventura A (2008) Is antibiotic prophylaxis in children with vesicoureteral reflux effective in preventing pyelonephritis and renal scars? A randomized, controlled trial. Pediatrics 121:e1489e1494

10. Roussey-Kesler G, Gadjos V, Idres N, Horen B, Ichay L, Leclair MD, Raymond F, Grellier A, Hazart I, De PL, Salomon R, Champion G, Leroy V, Guigonis V, Siret D, Palcoux JB, Taque S, Lemoigne A, Nguyen JM, Guyot C (2008) Antibiotic prophylaxis for the prevention of recurrent urinary tract infection in children with low grade vesicoureteral reflux: results from a prospective randomized study. J Urol 179:674-679 